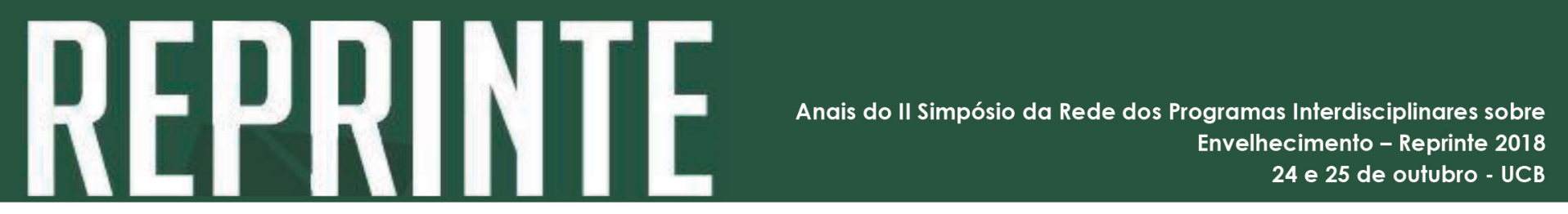

http://dx.doi.org/10.5335/rbceh.v16i1.9853

\title{
17) Aspectos fisiológicos e patológicos do envelhecimento tireoidiano no idoso: "tireoidopausa"
}

\author{
Laura de Lima Crivellaro'; Luan da Cruz Vieira'; Marina Ferreira da Silva'; \\ Kétuny da Silva Oliveira'; Fernanda Sousa Nascimento'; Fernanda Silveira Tavares²
}

\section{Resumo}

O atual debate sobre os aspectos fisiológicos e patológicos do envelhecimento tireoidiano no idoso levam ao processo de conscientizar médicos e pacientes acerca da importância do diagnóstico e tratamento precoce do hipotireoidismo em idosos, considerando sua prevalência, bem como da necessidade de valores referenciais mais específicos para a população idosa.

Palavras-chave: Hipotireoidismo; Tireoidopausa; Idosos; Tireoide; Envelhecimento.

\section{Introdução}

As disfunções tireoidianas apresentam-se como distúrbios endócrinos comuns em idosos. Isso se justifica pelo próprio envelhecimento e pelo aumento da prevalência de doenças tireoidianas nessa faixa etária. O hipotireoidismo é o distúrbio mais encontrado. Estima- se uma prevalência de $3,1 \%$ a 8,5\% na população geral, entretanto, o contingente tende a aumentar com a idade.

\section{Métodos}

Utilizou- se a base de dados Scielo, Pubmed e Google Acadêmico, nas quais foram selecionados 9 artigos de revisão publicados entre os anos de 2014 a 2018.

1 Graduandos em Medicina. Endereço para correspondência: QS 7, Lote 1, Águas Claras, Brasília - CEP: 71966-700, Brasília (DF), Brasil. E-mails: crivellarolimalaura@gmail.com; luanvieira96.Iv@gmail.com; marinaferreiraffs@gmail.com; ketunyoliveira@gmail.com; fsnascimento89@yahoo.com.br

2 Doutoranda no Programa de Pós-Graduação em Gerontologia, professora da disciplina de Endocrinologia do Curso de Medicina da Universidade Católica de Brasília-DF. Email: fernanda.endocrino@gmail.com 


\section{Resultados}

A apresentação clínica das doenças tireoidianas em idosos é inespecífica e as pessoas acometidas podem manifestar fadiga, ganho de peso, além de repercussões cardiovasculares (BARBESINO, 2018; CANNIZZARO et al, 2016). A elevada coexistência de outras doenças crônicas, os efeitos colaterais dos medicamentos e o próprio envelhecimento confundem o diagnóstico (CLEYDE et al, 2003; HENNESSEY; ESPAILLAT, 2015). Observa-se uma tendência à diminuição na produção de T3 e T4, definida pelo neologismo "tireoidopausa" (KANAAN et al, 2005; KLATZ.; GOLDMAN, 2006). A falta de diagnóstico ocasiona um aumento do risco cardiovascular, piora da função cognitiva e da qualidade de vida (ROMALDINI et al, 2004). Ainda assim, há aqueles que defendem uma prática mais conservadora para o hipotireoidismo primário e questionam um novo parâmetro de referência para o TSH em idosos (TAVARES, 1999). Obviamente, a abordagem deve ser individualizada, norteada por uma anamnese bem feita e contrabalanço entre prós e contras (TAVARES et, 2009).

\section{Conclusão}

Uma vez que os hormônios tireoidianos desempenham papel em praticamente todas as funções do organismo, e, considerando o envelhecimento populacional uma realidade, fica claro o quão e importante conhecer os marcadores e determinantes da "tireoidopausa", objetivando diferenciar os processos fisiológicos e patológicos e estabelecer diretrizes terapêuticas e referências laboratoriais mais adequadas a população idosa.

\section{Physiological and pathological aspects of thyroid aging in the elderly: "thyroid"}

\section{Abstract}

The current debate on the physiological and pathological aspects of aging in the elderly leads to the process of making doctors and patients aware of the importance of early diagnosis and treatment of hypothyroidism in the elderly, considering their prevalence, as well as the need for more specific reference values for the elderly. population.

Keywords: Hypothyroidism; Tireoidopause; Seniors; Thyroid; Aging.

\section{Referências}

BARBESINO, Giuseppe. Thyroid Function Changes in the Elderly and Their Relationship to Cardiovascular Health: A Mini-Review. Gerontology, p. 1-8, 2018.

CANNIZZARO, Matteo Angelo et al. The thyroid disease in the elderly: Our experience. International Journal of Surgery, v. 33, p. S85-S87, 2016.

CLYDE, Patrick W. et al. Combined levothyroxine plus liothyronine compared with levothyroxine alone in primary hypothyroidism: a randomized controlled trial. Jama, v. 290, n. 22, p. 2952-2958, 2003.

HENNESSEY, James V.; ESPAILLAT, Ramon. Diagnosis and management of subclinical hypothyroidism in elderly adults: a review of the literature. Journal of the American Geriatrics Society, v. 63, n. 8, p. 1663-1673, 2015. 
KANAAN, Salim; GARGIA, Maria Alice Terra; CARVALHO, Clayton Barbiéri de. Alterações laboratoriais hormonais em geriatria. J. bras. Med, v. 89, n. 5/6, p. 12-24, 2005.

KLATZ, R.; GOLDMAN, B. Anti-aging Therapeutics. Vol. VI. American Academy of Anti-Aging Medicine. Chicago, EEUU. 2006, pp. 9-10.

ROMALDINI, João Hamilton; SGARBI, José Augusto; FARAH, Chady Satt. Disfunções mínimas da tiróide: Hipotiroidismo subclínico e hipertiroidismo subclínico. Arq Bras Endocrinol Metabol, v. 48, n. 1, p. 147-158, 2004.

TAVARES, Adriano Bueno. Impacto do hipotireoidismo entre mulheres climatéricas. Revista Brasileira de Ginecologia e Obstetrícia, v. 21, n. 9, p. 560-560, 1999.

TAVARES, Fernanda Silveira et al. Tireoidoopausa: evidências e contra-evidências. Interciencia, 2009, 34.10. 